\title{
ERG oncoprotein expression in prostate carcinoma patients of different ethnicities
}

\author{
GREGORY M. KELLY ${ }^{1}$, YINK HEAY KONG ${ }^{1}$, ALBERT DOBI ${ }^{2}$, SHIV SRIVASTAVA ${ }^{2}$, ISABELL A. SESTERHENN ${ }^{3}$, \\ RAJADURAI PATHMANATHAN ${ }^{4}$, HUI MENG TAN ${ }^{4,5}$, SHYH-HAN TAN ${ }^{2}$ and SOK CHING CHEONG ${ }^{1}$ \\ ${ }^{1}$ Oral Cancer Research Team, Cancer Research Initiatives Foundation (CARIF), 2nd Floor Outpatient Centre, \\ Sime Darby Medical Centre, Subang Jaya, Selangor 47500, Malaysia; ${ }^{2}$ Department of Surgery, \\ Center for Prostate Disease Research, Uniformed Services University of the Health Sciences, Bethesda, MD 20814; \\ ${ }^{3}$ Joint Pathology Center, Silver Spring, MD 20910, USA; ${ }^{4}$ Sime Darby Medical Centre, Subang Jaya, Selangor 47500; \\ ${ }^{5}$ Faculty of Medicine, University of Malaya, Kuala Lumpur 50603, Malaysia
}

Received May 25, 2014; Accepted July 25, 2014

DOI: $10.3892 / \mathrm{mco} .2014 .418$

\begin{abstract}
Overexpression of the erythroblast transformation-specific-related gene (ERG) oncoprotein due to transmembrane protease, serine 2 (TMPRSS2)-ERG fusion, the most prevalent genomic alteration in prostate cancer $(\mathrm{CaP})$, is more frequently observed among Caucasian patients compared to patients of African or Asian descent. To the best of our knowledge, this is the first study to investigate the prevalence of $E R G$ alterations in a multiethnic cohort of $\mathrm{CaP}$ patients. A total of 191 formalin-fixed paraffin-embedded sections of transrectal ultrasound-guided prostate biopsy specimens, collected from 120 patients treated at the Sime Darby Medical Centre, Subang Jaya, Malaysia, were analyzed for ERG protein expression by immunohistochemistry using the anti-ERG monoclonal antibody 9FY as a surrogate for the detection of $E R G$ fusion events. The overall frequency of ERG protein expression in the population evaluated in this study was $39.2 \%$. Although seemingly similar to rates reported in other Asian communities, the expression of ERG was distinct amongst different ethnic groups $(\mathrm{P}=0.004)$. Malaysian Indian (MI) patients exhibited exceedingly high expression of ERG in their tumors, almost doubling that of Malaysian Chinese (MC) patients, whereas ERG expression was very low amongst Malay patients (12.5\%). When collectively analyzing data, we observed a significant correlation between younger patients and higher ERG expression ( $\mathrm{P}=0.04)$. The prevalence of $\mathrm{ERG}$ expression was significantly different amongst $\mathrm{CaP}$ patients of different ethnicities. The higher number of ERG-expressing
\end{abstract}

Correspondence to: Professor Sok Ching Cheong, Oral Cancer Research Team, Cancer Research Initiatives Foundation (CARIF), 2nd Floor Outpatient Centre, Sime Darby Medical Centre, 1 Jalan Ss12/1A, Subang Jaya, Selangor 47500, Malaysia

E-mail: sokching.cheong@carif.com.my

Key words: prostate cancer, transmembrane protease, serine 2-erythroblast transformation-specific-related gene fusion, Malaysia tumors among MI patients suggested that the TMPRSS2-ERG fusion may be particularly important in the pathogenesis of $\mathrm{CaP}$ amongst this group of patients. Furthermore, the more frequent expression of ERG among the younger patients analyzed suggested an involvement of ERG in the early onset of $\mathrm{CaP}$. The results of this study underline the value of using ERG status to better understand the differences in the etiology of $\mathrm{CaP}$ initiation and progression between ethnic groups.

\section{Introduction}

Prostate carcinoma $(\mathrm{CaP})$ is the fourth most frequently diagnosed cancer among Malaysian men, preceded by lung, colorectal and nasopharyngeal cancers (1). However, consistent with the global tendency of an increasing median age of patient populations due to increased overall longevity, the incidence of $\mathrm{CaP}$ in Malaysia is also on the increase (2). Among the major ethnic groups in Malaysia, the incidence of $\mathrm{CaP}$ was found to increase after the age of 45 years and is highest among Malaysian Chinese (MC) compared to Malaysian Indian (MI) and Malay men (1).

There is a clear disparity in $\mathrm{CaP}$ incidence and mortality worldwide and the major determining factors are being actively investigated. Although socioeconomic status and access to healthcare are often associated with disparities in the diagnosis, treatment and survival of $\mathrm{CaP}$ patients of different ethnic backgrounds, contributing genetic differences have also been identified $(3,4)$. Some of the tools used to characterize gene alterations and identify potential driver genes include genome-wide association studies, karyotyping of chromosomal copy number, as well as exome and whole-genome sequencing. In addition to the search for underlying genetic events that initiate cancer or distinguish aggressive from indolent tumors, the search for genetic alterations that may explain the ethnic disparities in $\mathrm{CaP}$ is currently actively pursued $(3,5,6)$.

The variant allele on $8 \mathrm{q} 24$, which increases the risk for $\mathrm{CaP}$, particularly in men of African ancestry, is one of the most convincing risk alleles for $\mathrm{CaP}(7,8)$. Another allele associated with an increased risk of $\mathrm{CaP}$ men of African ancestry is the rs743572 single-nucleotide polymorphism of CYP17 (9). 
More recently, evaluations of the transmembrane protease, serine 2 (TMPRSS2)-erythroblast transformation-specific (ETS)-related gene $(E R G)$ fusion in different populations have highlighted the differences in frequency between ethnic groups. Recurrent gene fusions between regulatory sequences of an androgen receptor (AR)-regulated gene, such as TMPRSS 2 , solute carrier family 45 member 3 or $\mathrm{N}$-myc downstream-regulated gene 1, and an ETS gene family member, such as $E R G, E T S$ translocation variant $(E T V) 1,4$ and 5 as the $3^{\prime}$ fusion partner, result in androgen-dependent expression of ETS transcription factors. Among these genetic alterations, the TMPRSS2-ERG fusion, detected in 50-70\% of $\mathrm{CaP}$ patients from Western countries, is the most prevalent $(10,11)$. The frequency of TMPRSS2-ERG gene fusions detected in CaPs of African Americans (AA) (31-43\%) is often lower compared to that of Caucasian Americans (CA) $(50-66 \%)(5,12)$. Interestingly, ERG overexpression is more frequently detected in the index tumors of CA (63.3\%), compared to those of AA patients (28.6\%) (5). However, evaluations of TMPRSS2-ERG gene fusions, either by immunohistochemistry (IHC) detection of ERG expression alone or in combination with fluorescence in situ hybridization (FISH), in different populations worldwide demonstrated lower frequencies compared to that detected CA and Europeans (12-20).

The aberrant overexpression of an ERG oncoprotein as a result of TMPRSS2-ERG fusion exerts a profound effect on cellular pathways associated with cancer initiation and progression (10,21-24). Evidence of the association between ERG-positive prostatic intraepithelial neoplasia lesions and ERG-positive prostate tumors highlights the significance of ERG activation in the early stages of tumor development (25). ERG overexpression inhibits prostate epithelial differentiation, while promoting epithelial-to-mesenchymal transition $(26,27)$. In addition, ERG regulates target genes with functions in DNA damage repair, epigenetic silencing and inflammation, which affect pathways associated with tumor cell growth, proliferation and invasion (24). For example, the cooperation of ERG with phosphatase and tensin homolog (PTEN) deletion and activation of AKT has been shown to promote neoplastic transformation $(28,29)$. A better understanding of how ERG interacts with cancer genes that contribute to cancer progression has led to the development of various treatment strategies that target ERG and its downstream effectors (30). The ability to clearly detect ERG expression in prostate tumors in contrast to normal glands by IHC using specific monoclonal antibodies (MAbs) has improved the diagnosis of the majority of CaPs $(25,31)$. The high concordance between the evaluations of TMPRSS2-ERG fusion by FISH and ERG protein expression by IHC supports the reliability and accuracy of ERG IHC as a surrogate for FISH detection (25,31-33). Furthermore, the evaluation of prostate tumors for ERG expression, together with PTEN deletion and integrity of AR signaling pathways, may help the prognostic stratification of patients and the selection of treatment options $(34,35)$.

To date, no study has evaluated the frequency of ERG alterations in $\mathrm{CaP}$ patients in Malaysia, which has a population comprising diverse ethnic groups. The major ethnic groups in Malaysia are Malays (55\%), Chinese (24\%) and Indians (7.2\%) (36). In order to better understand the role of ERG in the etiology of $\mathrm{CaP}$ initiation and progression, we used the
Table I. Demographics of prostate cancer patients $(n=120)$ and Gleason scores of tumor specimens $(n=191)$.

\begin{tabular}{lrr}
\hline Variables & No. & $\%$ \\
\hline Age (years) & & \\
$<69$ & 60 & 50.0 \\
$\geq 69$ & 60 & 50.0 \\
Ethnicity & & \\
Chinese & 82 & 68.3 \\
Malay & 8 & 6.7 \\
Indian & 30 & 25.0
\end{tabular}

Sections from right lobe $(\mathrm{n}=100)$

$\begin{array}{lll}\text { Gleason scores } & & \\ \leq 6 & 32 & 32.0 \\ 7(3+4) & 18 & 18.0 \\ 7(4+3), 8-10 & 50 & 50.0\end{array}$

Sections from left lobe $(n=91)$

Gleason scores

$\begin{array}{lll}\leq 6 & 27 & 29.7 \\ 7(3+4) & 20 & 22.0 \\ 7(4+3), 8-10 & 44 & 48.3\end{array}$

Total prostate sections $(\mathrm{n}=191)$

Total Gleason scores

$\begin{array}{lll}\leq 6 & 59 & 30.9\end{array}$

$\begin{array}{lll}7(3+4) & 38 & 19.9\end{array}$

$7(4+3), 8-10 \quad 94 \quad 49.2$

detection of ERG by IHC as a surrogate for $E R G$ fusion events to evaluate the prevalence of ERG expression in a multiethnic cohort of Malaysian CaP patients.

\section{Materials and methods}

Specimens. Transrectal ultrasound (TRUS)-guided biopsies were performed on 120 patients who were diagnosed with $\mathrm{CaP}$ based on clinical findings at the Sime Darby Medical Centre, Subang Jaya, Malaysia. The specimens were collected between 2011 and 2013, following approval by an independent Ethics Committee of Sime Darby Healthcare (ethics reference no. 201309.5). The TRUS-guided biopsy entails targeting the suspected prostatic lesion, as well as random sampling of the prostatic gland. Typically, 12-18 biopsy cores were collected from each prostate. Occasionally, 24-36 biopsy cores were collected from patients with significantly larger prostates, or from whom a second biopsy was required. The biopsy specimens were fixed with formalin and embedded in paraffin blocks.

IHC detection for ERG expression. Anti-ERG-MAb 9FY was obtained (cat. no. CM421C; Biocare Medical, Concord, CA, USA). Sections $(4-\mu \mathrm{m})$ were cut from formalin-fixed paraffin-embedded (FFPE) blocks, mounted on slides and deparaffinized. IHC was performed using a Ventana Benchmark Ultra autostainer (Ventana Medical Systems, Inc., Tucson, AZ, USA) using Ventana reagents. Briefly, the 


\begin{tabular}{|c|c|c|c|c|c|c|c|c|c|c|c|c|c|c|c|}
\hline No & Patient & Left Lobe & $\begin{array}{c}\text { Gleason } \\
\text { Sum (Left) }\end{array}$ & $\begin{array}{l}\text { Right } \\
\text { Lobe }\end{array}$ & $\begin{array}{c}\text { Gleason Sum } \\
\text { (Right) }\end{array}$ & $\begin{array}{c}\text { Overall ERG } \\
\text { Status }\end{array}$ & $\begin{array}{l}\text { Highest } \\
\text { Gleason }\end{array}$ & No & Patient & Left Lobe & $\begin{array}{c}\text { Gleason } \\
\text { Sum (Left) }\end{array}$ & $\begin{array}{l}\text { Right } \\
\text { Lobe }\end{array}$ & $\begin{array}{c}\text { Gleason Sum } \\
\text { (Right) }\end{array}$ & $\begin{array}{c}\text { Overall ERG } \\
\text { Status }\end{array}$ & $\begin{array}{l}\text { Highest } \\
\text { Gleason }\end{array}$ \\
\hline 1 & MC1 & & NS & & $3+3=6$ & - & 6 & 61 & MC61 & & $4+5=9$ & & $4+5=9$ & - & 9 \\
\hline 2 & MC2 & & NS & & $3+3=6$ & - & 6 & 62 & MC62 & & $5+4=9$ & & $5+4=9$ & - & 9 \\
\hline 3 & MC3 & & NS & & $3+3=6$ & - & 6 & 63 & MC63 & & $5+3=8$ & & $5+5=10$ & - & 10 \\
\hline 4 & MC4 & & NS & & $3+3=6$ & - & 6 & 64 & MC64 & & $3+3=6$ & & $3+3=6$ & + & 6 \\
\hline 5 & MCS & & NS & & $3+4=7$ & - & 7 & 65 & MC65 & & $3+3=6$ & & $4+5=9$ & + & 9 \\
\hline 6 & MC6 & & NS & & $3+4=7$ & - & 7 & 66 & MC66 & & $4+5=9$ & & $4+5=9$ & + & 9 \\
\hline 7 & MC7 & & NS & & $4+3=7$ & - & 7 & 67 & MC67 & & $2+3=5$ & & $2+3=5$ & + & 5 \\
\hline 8 & MC8 & & NS & & $4+4=8$ & - & 8 & 68 & MC68 & & $4+3=7$ & & $4+3=7$ & + & 7 \\
\hline 9 & MC9 & & NS & & $4+4=8$ & - & 8 & 69 & MC69 & & $3+4=7$ & & $3+4=7$ & + & 7 \\
\hline 10 & MC10 & & NS & & $4+5=9$ & - & 9 & 70 & MC70 & & $3+3=6$ & & $3+3=6$ & + & 6 \\
\hline 11 & MC11 & & NS & & $2+3=5$ & + & 5 & 71 & MC71 & & $3+3=6$ & & $3+3=6$ & + & 6 \\
\hline 12 & MC12 & & NS & & $3+3=6$ & + & 6 & 72 & MC72 & & $3+4=7$ & & $3+4=7$ & + & 7 \\
\hline 13 & MC13 & & NS & & $3+3=6$ & + & 6 & 73 & MC73 & & $4+4=8$ & & $4+4=8$ & + & 8 \\
\hline 14 & MC14 & & NS & & $4+3=7$ & + & 7 & 74 & MC74 & & $4+5=9$ & & $3+5=8$ & + & 9 \\
\hline 15 & MC15 & & NS & & $4+5=9$ & + & 9 & 75 & MC75 & & $3+3=6$ & & $3+3=6$ & + & 6 \\
\hline 16 & MC16 & & $3+3=6$ & & NS & - & 6 & 76 & MC76 & & $4+4=8$ & & $3+4=7$ & + & 8 \\
\hline 17 & MC17 & & $3+3=6$ & & NS & - & 6 & 77 & MC77 & & $4+5=9$ & & $4+5=9$ & + & 9 \\
\hline 18 & MC18 & & $3+3=6$ & & NS & - & 6 & 78 & MC78 & & $3+3=6$ & & $3+3=6$ & + & 6 \\
\hline 19 & MC19 & & $3+3=6$ & & NS & - & 6 & 79 & MC79 & & $3+4=7$ & & $3+3=6$ & + & 7 \\
\hline 20 & MC20 & & $3+4=7$ & & NS & - & 7 & 80 & MC80 & & $3+4=7$ & & $3+4=7$ & + & 7 \\
\hline 21 & MC21 & & $3+4=7$ & & NS & - & 7 & 81 & MC81 & & $4+4=8$ & & $4+4=8$ & + & 8 \\
\hline 22 & MC22 & & $3+4=7$ & & NS & - & 7 & 82 & MC82 & & $4+5=9$ & & $4+5=9$ & + & 9 \\
\hline 23 & MC23 & & $3+4=7$ & & NS & - & 7 & 83 & MI1 & & NS & & $3+3=6$ & - & 6 \\
\hline 24 & MC24 & & $4+3=7$ & & NS & - & 7 & 84 & M12 & & NS & & $3+3=6$ & - & 6 \\
\hline 25 & MC25 & & $4+4=8$ & & NS & - & 8 & 85 & M13 & & NS & & $3+3=6$ & - & 6 \\
\hline 26 & MC26 & & $4+4=8$ & & NS & - & 8 & 86 & M14 & & NS & & $3+4=7$ & - & 7 \\
\hline 27 & MC27 & & $3+3=6$ & & NS & + & 6 & 87 & MIS & & NS & & $5+4=9$ & - & 9 \\
\hline 28 & MC28 & & $3+3=6$ & & NS & + & 6 & 88 & M16 & & NS & & $4+5=9$ & - & 9 \\
\hline 29 & MC29 & & $3+4=7$ & & NS & + & 7 & 89 & MI7 & & NS & & $3+3=6$ & + & 6 \\
\hline 30 & MC30 & & $2+3=5$ & & $2+3=5$ & - & 5 & 90 & M18 & & NS & & $3+4=7$ & + & 7 \\
\hline 31 & MC31 & & $2+3=5$ & & $2+3=5$ & - & 5 & 91 & M19 & & NS & & $3+4=7$ & + & 7 \\
\hline 32 & MC32 & & $3+2=5$ & & $3+2=5$ & - & 5 & 92 & MI10 & & NS & & $3+4=7$ & + & 7 \\
\hline 33 & MC33 & & $2+3=5$ & & $2+3=5$ & - & 5 & 93 & MI11 & & NS & & $3+5=8$ & + & 8 \\
\hline 34 & MC34 & & $3+4=7$ & & $2+3=5$ & - & 7 & 94 & M112 & & NS & & $4+4=8$ & + & 8 \\
\hline 35 & MC35 & & $4+4=8$ & & $3+2=5$ & - & 8 & 95 & MI13 & & NS & & $4+5=9$ & + & 9 \\
\hline 36 & MC36 & & $3+3=6$ & & $3+3=6$ & - & 6 & 96 & MI14 & & $3+4=7$ & & NS & - & 7 \\
\hline 37 & MC37 & & $3+3=6$ & & $3+3=6$ & - & 6 & 97 & MI15 & & $3+3=6$ & & NS & + & 6 \\
\hline 38 & MC38 & & $3+3=6$ & & $3+3=6$ & - & 6 & 98 & MI16 & & $3+4=7$ & & NS & + & 7 \\
\hline 39 & MC39 & & $3+3=6$ & & $3+3=6$ & - & 6 & 99 & M!17 & & $4+3=7$ & & NS & + & 7 \\
\hline 40 & MC40 & & $3+3=6$ & & $3+3=6$ & - & 6 & 100 & M118 & & $3+4=7$ & & NS & + & 7 \\
\hline 41 & MC41 & & $3+3=6$ & & $3+3=6$ & - & 6 & 101 & M!19 & & $3+4=7$ & & $3+4=7$ & - & 7 \\
\hline 42 & MC42 & & $3+3=6$ & & $3+4=7$ & - & 7 & 102 & MI20 & & $4+4=8$ & & $4+4=8$ & - & 8 \\
\hline 43 & MC43 & & $3+4=7$ & & $3+4=7$ & - & 7 & 103 & M121 & & $4+4=8$ & & $4+4=8$ & - & 8 \\
\hline 44 & MC44 & & $4+3=7$ & & $4+3=7$ & - & 7 & 104 & M122 & & $5+3=8$ & & $5+4=9$ & - & 9 \\
\hline 45 & MC45 & & $3+4=7$ & & $3+4=7$ & - & 7 & 105 & M123 & & $3+4=7$ & & $3+4=7$ & + & 7 \\
\hline 46 & MC46 & & $3+4=7$ & & $3+4=7$ & - & 7 & 106 & M124 & & $4+3=7$ & & $4+3=7$ & + & 7 \\
\hline 47 & MC47 & & $4+4=8$ & & $4+4=8$ & - & 8 & 107 & M125 & & $4+5=9$ & & $4+5=9$ & + & 9 \\
\hline 48 & MC48 & & $4+4=8$ & & $4+4=8$ & - & 8 & 108 & M126 & & $3+4=7$ & & $3+3=6$ & + & 7 \\
\hline 49 & MC49 & & $4+4=8$ & & $4+4=8$ & - & 8 & 109 & M127 & & $5+4=9$ & & $3+4=7$ & + & 9 \\
\hline 50 & MC50 & & $4+4=8$ & & $4+4=8$ & - & 8 & 110 & M128 & & $5+4=9$ & & $5+4=9$ & + & 9 \\
\hline 51 & MC51 & & $3+3=6$ & & $5+4=9$ & - & 9 & 111 & M129 & & $4+5=9$ & & $4+5=9$ & + & 9 \\
\hline 52 & MC52 & & $5+4=9$ & & $5+4=9$ & - & 9 & 112 & MI30 & & $4+5=9$ & & $4+5=9$ & + & 9 \\
\hline 53 & MC53 & & $5+4=9$ & & $5+4=9$ & - & 9 & 113 & MAL1 & & NS & & $2+3=5$ & - & 5 \\
\hline 54 & MC54 & & $4+5=9$ & & $4+5=9$ & - & 9 & 114 & MAL2 & & $5+4=9$ & & NS & - & 9 \\
\hline 55 & MC55 & & $4+5=9$ & & $4+5=9$ & - & 9 & 115 & MAL3 & & $2+2=4$ & & $4+3=7$ & - & 7 \\
\hline 56 & MC56 & & $4+5=9$ & & $4+5=9$ & - & 9 & 116 & MAL4 & & $3+4=7$ & & $3+4=7$ & - & 7 \\
\hline 57 & MC57 & & $4+5=9$ & & $4+5=9$ & - & 9 & 117 & MAL5 & & $4+4=8$ & & $4+3=7$ & - & 8 \\
\hline 58 & MC58 & & $4+5=9$ & & $4+5=9$ & - & 9 & 118 & MAL6 & & $4+4=8$ & & $4+4=8$ & - & 8 \\
\hline 59 & MC59 & & $4+5=9$ & & $4+5=9$ & - & 9 & 119 & MAL7 & & $4+5=9$ & & $4+5=9$ & - & 9 \\
\hline 60 & MC60 & & $4+5=9$ & & 9 & - & 9 & 120 & MAL8 & & $4=9$ & & $4=9$ & + & 9 \\
\hline
\end{tabular}

Figure 1. Erythroblast transformation-specific-related gene (ERG) oncoprotein expression of tumor sections from each patient examined. Heatmap representation of ERG expression in both patients and sections. Green, no expression; pink, low expression; peach, moderate expression; dark red, strong expression. Grey, no sections evaluated. MC, Malaysian Chinese; NS, not scored; MI, Malaysian Indian; MAL, Malay.

sectioned specimens were processed for antigen retrieval using $\mathrm{CC} 1$ antigen retrieval solution prediluted in Tris/borate/EDTA buffer ( $\mathrm{pH} 8.0-8.5)$ and incubated at $95^{\circ} \mathrm{C}$ for $48 \mathrm{~min}$. The sections were then put through peroxidase inhibition prior to incubation with ERG-MAb at a dilution of 1:100 for 20 min at room temperature. ERG expression was detected by using OptiView HQ universal Linker and OptiView HRP Multimer (Ventana Medical Systems, Inc.), incubated consecutively at 
Table II. Association of erythroblast transformation-specific-related gene (ERG) oncoprotein expression status with ethnicity, age and Gleason score, as evaluated by patient and by individual tumor sections.

\begin{tabular}{|c|c|c|c|c|c|c|}
\hline \multirow[b]{3}{*}{ Variables } & \multicolumn{3}{|c|}{ Evaluation by patient $(\mathrm{n}=120)$} & \multicolumn{3}{|c|}{ Evaluation by individual tumor sections $(\mathrm{n}=191)$} \\
\hline & \multicolumn{2}{|c|}{ ERG expression } & \multirow[b]{2}{*}{ P-value } & \multicolumn{2}{|c|}{ ERG expression } & \multirow[b]{2}{*}{ P-value } \\
\hline & Negative (\%) & Positive (\%) & & Negative (\%) & Positive (\%) & \\
\hline Ethnicity & & & $0.004^{\mathrm{a}, \mathrm{b}}$ & & & $<0.001^{\mathrm{a}}$ \\
\hline Chinese & $55(67.1)$ & $27(32.9)$ & & $100(74.1)$ & $35(25.9)$ & \\
\hline Malay & $7(87.5)$ & $1(12.5)$ & & 13 (92.9) & $1(7.1)$ & \\
\hline Indian & $11(36.7)$ & $19(63.3)$ & & $18(42.9)$ & $24(57.1)$ & \\
\hline Total & $73(60.8)$ & $47(39.2)$ & & $131(68.6)$ & $60(31.4)$ & \\
\hline Age (years) & & & $0.040^{\mathrm{a}, \mathrm{c}}$ & & & $0.015^{\mathrm{a}, \mathrm{c}}$ \\
\hline$<69$ & $31(51.7)$ & $29(48.3)$ & & $56(60.2)$ & $37(39.8)$ & \\
\hline$\geq 69$ & $42(70.0)$ & $18(30.0)$ & & $75(76.5)$ & $23(23.5)$ & \\
\hline Gleason score & & & $0.813^{c}$ & & & $0.476^{\mathrm{c}}$ \\
\hline$\leq 6$ & $22(62.9)$ & $13(37.1)$ & & $41(69.5)$ & $18(30.5)$ & \\
\hline $7(3+4)$ & $15(55.6)$ & $12(44.4)$ & & $23(60.5)$ & $15(39.5)$ & \\
\hline $7(4+3), 8-10$ & $36(62.1)$ & $22(37.9)$ & & $67(71.3)$ & $27(28.7)$ & \\
\hline
\end{tabular}

${ }^{a} \mathrm{P}<0.05$ (statistically significant difference). Data were analyzed by ${ }^{\mathrm{b}}$ Kruskal-Wallis test or ${ }^{\mathrm{c}} \mathrm{Pearson}$ 's Chi-square test.
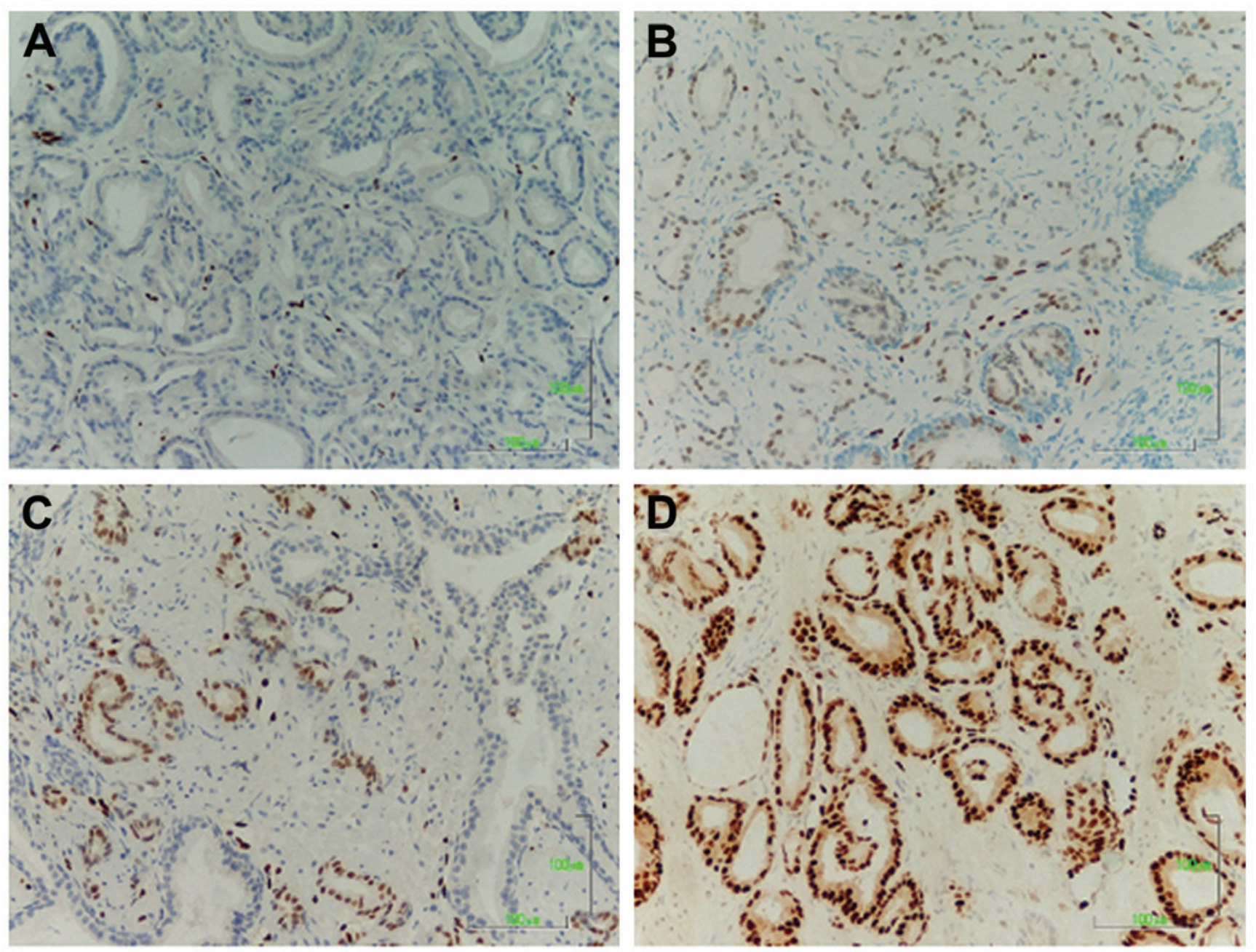

Figure 2. Immunohistochemical staining for the expression of erythroblast transformation-specific-related gene (ERG) oncoprotein using anti-ERG-MAb 9FY. Representative images showing (A) 0 , negative; (B) $1+$, mildly positive; (C) $2+$, moderately positive; and (D) $3+$, strongly positive staining for ERG expression. 
Table III. Association of erythroblast transformation-specific-related gene (ERG) oncoprotein staining intensity in the examined sections with ethnicity, age and Gleason score.

\begin{tabular}{lccc}
\hline & \multicolumn{3}{c}{ ERG staining intensity (\%) } \\
\cline { 2 - 4 } Variables & Negative (0) & Weak (1+) & Strong (2+ and 3+) \\
\hline Ethnicity & $100(74.1)$ & $7(5.2)$ & $28(20.7)$ \\
Chinese & $13(92.9)$ & $0(0.0)$ & $1(7.1)$ \\
Malay & $18(42.9)$ & $7(16.6)$ & $17(40.5)$ \\
Indian & & & $30(32.3)$ \\
Age (years) & $56(60.2)$ & $7(7.5)$ & $16(16.3)$ \\
$<69$ & $75(76.5)$ & $7(7.2)$ & $11(18.6)$ \\
$\geq 69$ & & & $13(34.2)$ \\
Gleason score & $41(69.5)$ & $7(11.9)$ & $22(23.4)$ \\
$\leq 6$ & $23(60.5)$ & $2(5.3)$ & $0.032^{\mathrm{a}, \mathrm{c}}$ \\
$7(3+4)$ & $67(71.3)$ & $5(5.3)$ & \\
$7(4+3), 8-10$ & & & \\
\hline
\end{tabular}

${ }^{\mathrm{a}} \mathrm{P}<0.05$ (statistically significant difference). Data were analyzed by ${ }^{\mathrm{b}}$ Kruskal-Wallis test or ${ }^{\mathrm{c}} \mathrm{Pearson}$ 's Chi-square test.

room temperature for $8 \mathrm{~min}$. The color was developed using Bluing reagent for 4 min and the sections were counterstained with hematoxylin. The ERG protein expression status and Gleason scores of the prostate sections were evaluated by a trained pathologist. Depending on the amount and intensity of the ERG IHC staining, the specimens were scored as follows: 0 , negative; $1+$, mild; $2+$, moderate; and $3+$, strong staining. Positive staining of endothelial cells in the specimens served as a built-in control for the staining.

Statistical analysis. Statistical analyses were performed using SPSS 16.0 software for Windows (IBM, Inc., New York, NY, USA). The Pearson's Chi-square and Kruskal-Wallis tests were used to determine the statistical associations of ERG expression with ethnicity, age and Gleason sum score. $\mathrm{P}<0.05$ was considered to indicate a statistically significant difference.

\section{Results}

Patient demographics and Gleason score of prostate specimens. The ERG oncoprotein expression status was evaluated in tumor specimens from 120 patients by demographic distribution and tumor Gleason scores (Table I). The mean age of the patients was 69 years (range, 52-91 years). MC patients represented the largest ethnic group in this study (68.3\%), followed by MI $(25 \%)$ and Malay patients $(6.7 \%)$. Both the left and right lobes of the prostate were biopsied in all the patients. Among the 120 patients, 71 were found to have tumors in both lobes, whereas 20 patients had tumors in only the left lobe and 29 had tumors in only the right lobe of the prostate. Specifically, of the 191 tumor sections that were evaluated, 91 were from the left lobe and 100 were from the right lobe of the prostate (Table I and Fig. 1). The ERG expression status for each patient was scored as positive if ERG oncoprotein expression was detected in tumor sections from either lobe, taking into account inter-tumoral heterogeneity within the same prostate (25).
Prevalence of ERG expression in different ethnic groups. The evaluation of ERG oncoprotein expression by IHC in the multiethnic cohort of Malaysian CaP patients revealed an overall frequency of $39.2 \%$, with positive ERG expression in 47 of the 120 patients. ERG-positive tumors were detected in $31.4 \%(60 / 191)$ of the individual tumor sections examined. The status and intensity of ERG staining are detailed in the heatmap in Fig. 1 and sections representative for each level of expression are shown in Fig. 2.

Among the MC patients, who formed the majority ethnic group of this study, 27 of 82 cases (32.9\%) were ERG-positive (Table II). Prostate tumor sections were evaluated from either the right or left lobe of the prostate in 29 cases and from both lobes in 53 cases (Fig. 1). Positive ERG expression was detected in 35 of the 135 sections (25.9\%) examined (Table II). Of the 27 cases positive for ERG expression, ERG was detected in either the right or the left lobe of the prostate in 19 and in both lobes in 8 cases.

Surprisingly, 19 of 30 (63.3\%) MI patients were positive for ERG expression (Table II). Biopsy specimens were examined from either the right or left lobe of the prostate in 18 and from both lobes in 12 cases. Positive ERG expression was detected in 24 of 42 (57.1\%) individual tumor sections (Table II). Of the 19 MI patients with a positive ERG expression status, ERG was detected in either the right or the left lobe of the prostate in 14 patients and in both lobes in 5 cases.

Among the 8 Malay patients evaluated, only 1 (12.5\%) was positive for ERG (Table II). Prostate tumor sections from both lobes of the prostate were evaluated for 6 of the 8 Malay patients. Only 1 of the $14(7.1 \%)$ sections examined was positive for ERG expression (Table II).

Analysis of the association of the ERG expression status of patients with age and Gleason score. The association of ERG expression status of patients with age and Gleason score of tumors was evaluated by statistical analysis. The 
Table IV. Summary of the frequency of erythroblast transformation-specific-related gene (ERG) oncoprotein expression status in different populations worldwide.

\begin{tabular}{|c|c|c|c|c|}
\hline Population & Sample & $\begin{array}{l}\text { Assay method } \\
\text { for ERG detection }\end{array}$ & $\begin{array}{l}\text { Frequency, \% } \\
\quad \text { (no./total) }\end{array}$ & (Refs.) \\
\hline \multicolumn{5}{|l|}{ USA } \\
\hline NSeg & $\mathrm{RP}$ & FISH & $41.6(217 / 521)$ & $(45)$ \\
\hline $\mathrm{CA}$ & Biopsy & FISH & $46.0(46 / 100)$ & $(46)$ \\
\hline NSeg & $\mathrm{RP}, \mathrm{WM}$ & IHC, FISH & $65.1(86 / 132)$ & $(25)$ \\
\hline CA & $\mathrm{RP}$ & FISH & $50.0(21 / 42)$ & $(12)$ \\
\hline AA & $\mathrm{RP}$ & FISH & $31.3(20 / 64)$ & $(12)$ \\
\hline $\mathrm{CA}$ & $\mathrm{RP}, \mathrm{WM}$ & IHC, FISH & $65.9(60 / 91)$ & (5) \\
\hline AA & $\mathrm{RP}, \mathrm{WM}$ & IHC, FISH & $42.9(39 / 91)$ & (5) \\
\hline UK & TURP & FISH & $30.1(134 / 445)$ & (37) \\
\hline \multirow[t]{2}{*}{ Sweden } & TURP & FISH, RT-PCR & $17.5(62 / 354)$ & $(47)$ \\
\hline & TURP & FISH, RT-PCR & $16.9(46 / 272)$ & $(48)$ \\
\hline \multirow[t]{2}{*}{ Germany } & PCa, LNMets, Mets & IHC, FISH & $45.3(120 / 265)$ & $(32)$ \\
\hline & RP & FISH & $58.7(44 / 75)$ & $(49)$ \\
\hline \multirow[t]{4}{*}{ Japan } & $\mathrm{RP}$ & FISH & $15.9(7 / 44)$ & (12) \\
\hline & $\mathrm{RP}$ & IHC & $16.3(15 / 92)$ & $(17)$ \\
\hline & RP and biopsy & IHC & $20.1(42 / 209)$ & $(16)$ \\
\hline & $\mathrm{RP}$ & RT-PCR & $27.8(54 / 194)$ & $(15)$ \\
\hline \multirow[t]{2}{*}{ Korea } & $\mathrm{RP}$ & FISH & $20.9(53 / 254)$ & (13) \\
\hline & $\mathrm{RP}$ & IHC & $24.4(73 / 303)$ & (18) \\
\hline \multirow[t]{3}{*}{ China } & NS & FISH & $7.5(7 / 93)$ & (14) \\
\hline & & IHC & $10.2(9 / 88)$ & $(50)$ \\
\hline & TURP & FISH & $23.2(44 / 190)$ & $(20)$ \\
\hline India & $\mathrm{RP}$ & IHC, FISH & $26.7(8 / 30)$ & (19) \\
\hline \multicolumn{5}{|l|}{ Malaysia } \\
\hline NSeg & TRUS-biopsy & IHC & $39.2(47 / 120)$ & Present study \\
\hline $\mathrm{MC}$ & & & $32.9(27 / 82)$ & \\
\hline MI & & & $63.3(19 / 30)$ & \\
\hline Malay & & & $12.5(1 / 8)$ & \\
\hline
\end{tabular}

NS, not specified. NSeg, not segregated; RP, radical prostatectomy; FISH, fluorescence in situ hybridization; CA, Caucasian American; WM, whole-mounted prostate sections; IHC, immunohistochemistry; AA, African American; TURP, transurethral resection of the prostate; RT-PCR, reverse transcription-polymerase chain reaction; PCa, localized prostate cancer; LNMets, lymph node metastasis; Mets, metastasis; TRUS, transrectal ultrasound; MC, Malaysian Chinese; MI, Malaysian Indian.

results revealed a positive correlation between positive ERG expression of tumors and younger patients, when evaluated either by patient $(\mathrm{P}=0.04)$ or by individual tumor sections ( $\mathrm{P}=0.015$; Table II). We also observed a correlation between higher intensity of ERG staining with younger patients as a whole ( $\mathrm{P}=0.032$; Table III). The evaluation of the association between ERG expression status and Gleason score, either by patient or by individual tumor sections, did not reveal a significant correlation (Table II).

\section{Discussion}

In this study, we evaluated the expression of ERG oncoprotein in a multiethnic cohort of patients as a surrogate for the detection of TMPRSS2-ERG fusion events. We examined 191 sections of FFPE prostate tumor specimens isolated by TRUS-guided biopsy from 120 patients. The ethnic distribution of this study cohort, which consisted of $82 \mathrm{MC}$ (68.3\%), $30 \mathrm{MI}$ (25.0\%) and 8 Malay men $(6.7 \%)$, is representative of patient enrollment at the hospital where this study was conducted and does not mirror the ethnic distribution of the overall Malaysian population. However, it does represent the overall incidence of $\mathrm{CaP}$ diagnosed in the country, with the highest incidence among MC, followed by MI, and the lowest among Malays (1).

The overall frequency of ERG oncoprotein expression in the cohort of Malaysian CaP patients, as determined by IHC, was 39.2\%, which was considerably lower compared to the frequency of $50-70 \%$ detected in Western countries. The prevalence of ERG among MC, the largest ethnic group analyzed, was $32.9 \%$. Although this frequency of 
ERG-positive expression in the MC population is marginally higher compared to the frequencies of 15.9-29.7\% reported for populations from Korea, Japan and China, it remains within a similar range $(12,14,15,18,20)$ (Table IV).

Interestingly, we detected a disproportionately higher frequency (63.3\%) of ERG-positive tumors among MI patients in this study. This is in comparison to a previous study on Indian $\mathrm{CaP}$ patients without prior hormonal treatment from New Delhi, India, in which ERG-positive tumors were detected in 8 of the 30 cases (27\%) examined (19). However, the higher prevalence of ERG-positive cases in this study may be attributed to the limitations inherent in a small sample size. Whether the higher prevalence of ERG-positive tumors among MI patients indicates a regional variation where TMPRSS2-ERG fusion contributes more significantly to the progression of the disease compared to other populations of the same ethnicity, requires confirmation by studies on larger populations. Among the three ethnic groups, Malay $\mathrm{CaP}$ patients exhibited the lowest frequency (12.5\%) of ERG-positive tumors. However, the results obtained from the small sample of Malay patients analyzed in this study require further confirmation in studies involving larger cohorts.

Efforts to identify the correlation of TMPRSS2-ERG fusion or ERG overexpression with clinicopathological characteristics have yielded variable results, which is likely due to the heterogeneity of patient cohorts evaluated in different studies. In certain studies, a higher Gleason score and a lower tumor differentiation exhibited a significant correlation with ERG gene alterations or with ERG-positive immunostaining (25,37-39). Other studies have reported the association of a lower Gleason score with a higher number of TMPRSS2-ERG fusion events $(13,40)$. However, other studies have reported a significant association of TMPRSS2-ERG fusion with tumors of higher stage and lymph node metastasis (41) or higher pathological stage (42), but no association between TMPRSS2-ERG fusion and Gleason score. In a comparison between patients of different ethnic backgrounds, Rosen et al (5) reported a correlation between ERG-negative status and high-grade CaP tumors among AA but not among CA patients. There was no significant correlation between Gleason score and ERG expression or intensity when evaluated against either tumor sections or patients in our study. However, a significant association between younger patients (aged $<69$ years) and a positive ERG expression status, as well as ERG intensity, was observed in our Malaysian cohort as a whole. This correlation was also observed in studies among Japanese and European CaP patients $(17,43)$, which suggests that ERG rearrangement may be particularly important in patients with early-onset $\mathrm{CaP}$.

The effect of multiple factors, including diet, genetics and environmental factors, may contribute to the significant disparity in the frequency of CaP globally. The TMPRSS2-ERG gene fusion alteration, which is frequent among Western Caucasian populations, has been found to be less frequent among South Asian and East Asian populations. Whether other genomic alteration events typified by the fusion of other ETS gene family members, such as ETV1 and ETV4, to androgen-regulated promoters $(10,23)$, amplification of the $8 \mathrm{q} 24$ loci (44), PTEN deletion (20), or yet to be identified genetic events, are more prevalent in Asian populations remains to be investigated. A more comprehensive study, including a larger number of Malay and MI patients should be undertaken, not only to confirm the frequency of TMPRSS2-ERG fusion events, but to gain better understanding of the underlying genetics of $\mathrm{CaP}$ in the Malaysian population.

\section{Acknowledgements}

This study was funded by the sponsors of the Cancer Research Initiatives Foundation (CARIF). We are grateful to Mr. Vijaya Kumar T. Krishnan and Ms. Mary Catherine D. Cruz from the Histopathology Laboratory, Sime Darby Medical Centre, for their technical assistance. The Henry M. Jackson Foundation for the Advancement of Military Medicine has filed a patent application on the mouse monoclonal anti-ERG antibody, 9FY, on which S.T., A.D. and S.S. are co-inventors and have been licensed to the Biocare Medical. This study was conducted independently of any involvement from Biocare Medical.

\section{References}

1. Zainal Ariffin O and Nor Saleha IT: National Cancer Registry Report 2007. Malaysia Cancer Statistics - Data and Figure. National Cancer Registry, Ministry of Health, Putrajaya, Malaysia, pp, 2011.

2. Othman NH, Nor ZM and Biswal BM: Is Kelantan joining the global cancer epidemic? - experience from hospital Universiti Sains Malaysia; 1987-2007. Asian Pac J Cancer Prev 9: 473-478, 2008.

3. Farrell J, Petrovics G, McLeod DG and Srivastava S: Genetic and molecular differences in prostate carcinogenesis between African American and Caucasian American men. Int J Mol Sci 14: 15510-15531, 2013.

4. Mononen N and Schleutker J: Polymorphisms in genes involved in androgen pathways as risk factors for prostate cancer. J Urol 181: 1541-1549, 2009.

5. Rosen P, Pfister D, Young D, et al: Differences in frequency of ERG oncoprotein expression between index tumors of Caucasian and African American patients with prostate cancer. Urology 80: 749-753, 2012.

6. Martin DN, Starks AM and Ambs S: Biological determinants of health disparities in prostate cancer. Curr Opin Oncol 25: 235-241, 2013.

7. Amundadottir LT, Sulem P, Gudmundsson J, et al: A common variant associated with prostate cancer in European and African populations. Nat Genet 38: 652-658, 2006.

8. Freedman ML, Haiman CA, Patterson N, et al: Admixture mapping identifies $8 \mathrm{q} 24$ as a prostate cancer risk locus in African-American men. Proc Natl Acad Sci USA 103: 14068-14073, 2006.

9. Taioli E, Sears V, Watson A, et al: Polymorphisms in CYP17 and CYP3A4 and prostate cancer in men of African descent. Prostate 73: 668-676, 2013.

10. Rubin MA, Maher CA and Chinnaiyan AM: Common gene rearrangements in prostate cancer. J Clin Oncol 29: 3659-3668, 2011.

11. Rosen P, Sesterhenn IA, Brassell SA, McLeod DG, Srivastava S and Dobi A: Clinical potential of the ERG oncoprotein in prostate cancer. Nat Rev Urol 9: 131-137, 2012.

12. Magi-Galluzzi C, Tsusuki T, Elson P, et al: TMPRSS2-ERG gene fusion prevalence and class are significantly different in prostate cancer of Caucasian, African-American and Japanese patients. Prostate 71: 489-497, 2011.

13. Lee K, Chae JY, Kwak C, Ku JH and Moon KC: TMPRSS2-ERG gene fusion and clinicopathologic characteristics of Korean prostate cancer patients. Urology 76: 1268.e7-e13, 2010.

14. Mao X, Yu Y, Boyd LK, et al: Distinct genomic alterations in prostate cancers in Chinese and Western populations suggest alternative pathways of prostate carcinogenesis. Cancer Res 70: 5207-5212, 2010.

15. Miyagi Y, Sasaki T, Fujinami K, et al: ETS family-associated gene fusions in Japanese prostate cancer: analysis of 194 radical prostatectomy samples. Mod Pathol 23: 1492-1498, 2010. 
16. Furusato B, van Leenders GJ, Trapman J, et al: Immunohistochemical ETS-related gene detection in a Japanese prostate cancer cohort: diagnostic use in Japanese prostate cancer patients. Pathol Int 61: 409-414, 2011.

17. Kimura T, Furusato B, Miki J, et al: Expression of ERG oncoprotein is associated with a less aggressive tumor phenotype in Japanese prostate cancer patients. Pathol Int 62: 742-748, 2012.

18. Suh JH, Park JW, Lee C and Moon KC: ERG immunohistochemistry and clinicopathologic characteristics in Korean prostate adenocarcinoma patients. Korean J Pathol 46: 423-428, 2012.

19. Rawal S, Young D, Williams M, et al: Low frequency of the ERG oncogene alterations in prostate cancer patients from India. J Cancer 4: 468-472, 2013.

20. Qi M, Yang X, Zhang F, et al: ERG rearrangement is associated with prostate cancer-related death in Chinese prostate cancer patients. PLoS One 9: e84959, 2014.

21. Petrovics G, Liu A, Shaheduzzaman S, et al: Frequent overexpression of ETS-related gene-1 (ERG1) in prostate cancer transcriptome. Oncogene 24: 3847-3852, 2005.

22. Tomlins SA, Rhodes DR, Perner S, et al: Recurrent fusion of TMPRSS2 and ETS transcription factor genes in prostate cancer. Science 310: 644-648, 2005.

23. Dobi A, Sreenath T and Srivastava S: Androgen-dependent oncogenic activation of ETS transcription factors by recurrent gene fusions in prostate cancer: biological and clinical implications. In: Androgen-Responsive Genes in Prostate Cancer. Wang Z (ed). Springer, New York, NY, pp307-328, 2013.

24. Sreenath TL, Dobi A, Petrovics G and Srivastava S: Oncogenic activation of ERG: a predominant mechanism in prostate cancer. J Carcinog 10: 37, 2011.

25. Furusato B, Tan SH, Young D, et al: ERG oncoprotein expression in prostate cancer: clonal progression of ERG-positive tumor cells and potential for ERG-based stratification. Prostate Cancer Prostatic Dis 13: 228-237, 2010.

26. Sun C, Dobi A, Mohamed A, et al: TMPRSS2-ERG fusion, a common genomic alteration in prostate cancer activates C-MYC and abrogates prostate epithelial differentiation. Oncogene 27 : 5348-5353, 2008.

27. Gupta S, Iljin K, Sara H, et al: FZD4 as a mediator of ERG oncogene-induced WNT signaling and epithelial-to-mesenchymal transition in human prostate cancer cells. Cancer Res 70 6735-6745, 2010

28. Carver BS, Tran J, Gopalan A, et al: Aberrant ERG expression cooperates with loss of PTEN to promote cancer progression in the prostate. Nat Genet 41: 619-624, 2009.

29. King JC, Xu J, Wongvipat J, et al: Cooperativity of TMPRSS2-ERG with PI3-kinase pathway activation in prostate oncogenesis. Nat Genet 41: 524-526, 2009.

30. Rahim S and Uren A: Emergence of ETS transcription factors as diagnostic tools and therapeutic targets in prostate cancer. Am J Transl Res 5: 254-268, 2013

31. Park K, Tomlins SA, Mudaliar KM, et al: Antibody-based detection of ERG rearrangement-positive prostate cancer. Neoplasia 12: 590-598, 2010.

32. Braun M, Goltz D, Shaikhibrahim Z, et al: ERG protein expression and genomic rearrangement status in primary and metastatic prostate cancer - a comparative study of two monoclonal antibodies. Prostate Cancer Prostatic Dis 15: 165-169, 2012.
33. Svensson MA, Perner S, Ohlson AL, et al: A comparative study of ERG status assessment on DNA, mRNA, and protein levels using unique samples from a Swedish biopsy cohort. Appl Immunohistochem Mol Morphol 22: 136-141, 2014.

34. Gsponer JR, Braun M, Scheble VJ, et al: ERG rearrangement and protein expression in the progression to castration-resistant prostate cancer. Prostate Cancer Prostatic Dis 17: 126-131, 2014.

35. Fontugne J, Lee D, Cantaloni C, et al: Recurrent prostate cancer genomic alterations predict response to brachytherapy treatment. Cancer Epidemiol Biomarkers Prev 23: 594-600, 2014.

36. Hasan ARb: Statistical Handbook Malaysia 2013. Department of Statistics, Malaysia, 2013.

37. Attard G, Clark J, Ambroisine L, et al: Duplication of the fusion of TMPRSS2 to ERG sequences identifies fatal human prostate cancer. Oncogene 27: 253-263, 2008.

38. Rajput AB, Miller MA, De Luca A, et al: Frequency of the TMPRSS2:ERG gene fusion is increased in moderate to poorly differentiated prostate cancers. J Clin Pathol 60: 1238-1243, 2007.

39. Demichelis F, Fall K, Perner S, et al: TMPRSS2:ERG gene fusion associated with lethal prostate cancer in a watchful waiting cohort. Oncogene 26: 4596-4599, 2007.

40. Darnel AD, Lafargue CJ, Vollmer RT, Corcos J and Bismar TA: TMPRSS2-ERG fusion is frequently observed in Gleason pattern 3 prostate cancer in a Canadian cohort. Cancer Biol Ther 8: 125-130, 2009.

41. Perner S, Demichelis F, Beroukhim R, et al: TMPRSS2:ERG fusion-associated deletions provide insight into the heterogeneity of prostate cancer. Cancer Res 66: 8337-8341, 2006.

42. Mehra R, Tomlins SA, Shen R, et al: Comprehensive assessment of TMPRSS2 and ETS family gene aberrations in clinically localized prostate cancer. Mod Pathol 20: 538-544, 2007.

43. Schaefer G, Mosquera JM, Ramoner R, et al: Distinct ERG rearrangement prevalence in prostate cancer: higher frequency in young age and in low PSA prostate cancer. Prostate Cancer Prostatic Dis 16: 132-138, 2013.

44. Fromont G, Godet J, Peyret A, et al: 8q24 amplification is associated with Myc expression and prostate cancer progression and is an independent predictor of recurrence after radical prostatectomy. Hum Pathol 44: 1617-1623, 2013.

45. Gopalan A, Leversha MA, Satagopan JM, et al: TMPRSS2-ERG gene fusion is not associated with outcome in patients treated by prostatectomy. Cancer Res 69: 1400-1406, 2009.

46. Mosquera JM, Mehra R, Regan MM, et al: Prevalence of TMPRSS2-ERG fusion prostate cancer among men undergoing prostate biopsy in the United States. Clin Cancer Res 15: 4706-4711, 2009.

47. Setlur SR, Mertz KD, Hoshida Y, et al: Estrogen-dependent signaling in a molecularly distinct subclass of aggressive prostate cancer. J Natl Cancer Inst 100: 815-825, 2008.

48. Sboner A, Demichelis F, Calza S, et al: Molecular sampling of prostate cancer: a dilemma for predicting disease progression. BMC Med Genomics 3: 8, 2010.

49. Hofer MD, Kuefer R, Maier C, et al: Genome-wide linkage analysis of TMPRSS2-ERG fusion in familial prostate cancer. Cancer Res 69: 640-646, 2009.

50. Xue L, Mao X, Ren G, et al: Chinese and Western prostate cancers show alternate pathogenetic pathways in association with ERG status. Am J Cancer Res 2: 736-744, 2012. 